\title{
Prevalence of Chronic Malnutrition (Stunting) and Determinant Factors among Children Aged 0-23 Months in Western Ethiopia: A Cross-Sectional Study
}

\section{Tsedeke Wolde*, Emiru Adeba and Alemu Sufa}

Department of Public Health, College of Medical and Health Sciences, Wollega University, P.O.Box: 395, Nekemte, Ethiopia

*Corresponding author: Tsedeke Wolde, Department of Public Health, College of Medical and Health Sciences, Wollega University, Nekemte, Ethiopia, P.O.Box: 395, Tel: +251910943969; E-mail: tsedekewolde@yahoo.com

Received date: Jul 31, 2014, Accepted date: Sep 24, 2014, published date: Oct 1, 2014

Copyright: (c) 2014 Wolde T, et al. This is an open-access article distributed under the terms of the Creative Commons Attribution License, which permits unrestricted use, distribution, and reproduction in any medium, provided the original author and source are credited.

\begin{abstract}
Introduction: Poor growth especially stunting is associated with impaired development which is apparent in the relationship between growth status and school performance and intellectual achievement. Thus, previous studies in Western Ethiopia were not addressed factors associated with stunting.
\end{abstract}

Objective: To assess prevalence and determinants of stunting among less than 24 months children in East Wollega Zone, West Ethiopia.

Methods: A community based cross-sectional study design using two-stage cluster sampling survey was conducted on 593 households from April to May, 2014 in three randomly selected districts of East Wollega Zone to assess factors associated with stunting. A structured and pre-tested questionnaire was used to obtain information on demographic and socio economics characteristics, feeding practices, dietary diversity and anthropometric measurement of children aged less than two years. Bivariate and multivariable logistic regression models were fit to identify significant predictors of stunting at $\mathrm{P}<0.05$.

Results: Prevalence of stunting and severe stunting were $15.7 \%(95 \% \mathrm{Cl}: 12.7-18.7)$ and $0.3 \%(95 \% \mathrm{Cl}$ : 0.1-0.5) for children aged $<24$ months. Stunting was associated with illiterate mothers (AOR $=3.84 ; 95 \% \mathrm{Cl} 1.49-9.91)$ and nonexclusive breast feeding $(\mathrm{AOR}=2.12 ; 95 \% \mathrm{Cl} 1.19-7.79)$. Children who consumed vegetables and fruits $(\mathrm{AOR}=$ $0.51 ; 95 \% \mathrm{Cl} 0.28-0.95)$ and boiling drinking water $(\mathrm{AOR}=0.61,95 \% \mathrm{Cl}: 0.39-0.97)$ were significantly reduced odds of being stunted.

Conclusion and recommendation: The prevalence rate of stunting in the study area was found low. Stunting was significantly associated with the illiterate mothers and non-exclusive breastfeeding practice. Thus, an organized effort should be made at all levels to improve maternal education and exclusive breastfeeding practice of the poor rural population particularly mothers to curb the problems of chronic undernutrition (stunting) in children, especially in the first two years of life.

Keywords: Determinant factors; Stunting; Under 2 Children; Western Ethiopia

\section{Introduction}

Globally, it is estimated that under nutrition is responsible, directly or indirectly, for at least $35 \%$ of deaths in children less than five years of age. Under nutrition is also a major cause of disability preventing children who survive from reaching their full developmental potential. Stunting (deficit in height for age of at least $-2 \mathrm{z}$ score) affects close to 195 million children under five years of age in the developing world [1].

The period from birth to two years of age is particularly important because of the rapid growth and brain development that occurs during this time. The period is often marked by growth faltering, micronutrient deficiencies and common childhood illnesses such as diarrhea, as children transition from exclusive breastfeeding to solid foods in addition to breast milk [2]. Poor growth especially stunting is associated with impaired development which is apparent in the relationship between growth status, school performance and intellectual achievement [3].

Data exists in Ethiopia that show the problem of malnutrition beginning early in life, primarily during the first 12 months when growth faltering takes hold due to sub-optimal infant feeding practices. Once this growth faltering occurs, there is little opportunity for catch-up growth. Stunted infants grow to be stunted children and stunted adults. Thus, it is important to address issues of infant feeding during the first year of life, particularly promoting proven optimal breastfeeding and complementary feeding practices, both in healthy as well as sick infants [4]. At national level, 44 percent of children under age five are stunted and 21 percent of children are severely stunted. In Oromia region, prevalence of stunting children is $41.4 \%$ [5].

This paper assesses the prevalence and determinant factors associated with stunting among children aged 0-23 months old in Western Ethiopia. Therefore, to attain the already set goals in the country it is sound full to assess the association of stunting and some of the main determinant factors that have impacts on child feeding in the study area. The result of the study was benefit policy maker by 
providing appropriate information in order to create appropriate infant and young child feeding policy.

\section{Methods}

\section{Study setting, design and participants}

The study area was carried out in East Wollega Zone, Arjo Jimma, Sibu Sire and Arjo Gudetu districts, Oromia regional state, Western Ethiopia, which is located at $331 \mathrm{~km}$ from the capital Addis Ababa. The total population of East Wollega Zone is estimated to be $1,230,402$; out of this 615,641 are females according to the 2007 population and household survey. Eighty six percent of the population leaves in rural areas $(1,061,120)$. The Zone was divided into 21 (administrative districts) [7]. The dominant religion is Protestant Christianity followed by Orthodox.

The study period was conducted from April to May, 2014. A community based cross sectional study design was employed in the three rural districts community of East Wollega Zone, Western Ethiopia. The study population was all mothers of children aged 0 to 23 months.

The sample size for this study was determined using a formula for estimation of single population proportion assuming an expected prevalence for stunted children in rural part of Ethiopia 46\%, $95 \%$ confidence level, $5 \%$ margin of error, a design effect of 1.5 and a nonresponse rate of 5\% [5]. A total of 602 mother-child pairs were identified using a two stage cluster sampling technique from the rural residence. Then, a census was conducted to get the sampling frame for selecting mother-child pairs by simple random sampling technique. Eligibility criteria were selected mother-child pairs who have permanent residence in the study area having apparently healthy children from 0 to 23 months old. An exclusion criterion was a child with evidence of physical impairment (such as physical defects or a grossly deformed), mental impairment and edematous conditions.

\section{Measurements}

Quantitative data were collected using a validated questionnaire adapted from the Ethiopian Health and Demo-graphic Survey (EDHS) [5]. The questionnaire covered a range of topics including: Socioeconomic and demographic variables; ethnicity, religion, household family size, wealth index, education (paternal/maternal) and occupation; Child characteristics; Age, sex, birth order, birth weight, boiling water, dietary diversity, breastfeeding and complementary feeding status and Maternal characteristics; age, number of children ever born, Antenatal Care (ANC) visits, advice/health information after delivery were collected by interviewing the mothers of index children.

Age of the child was calculated both from the child's date of birth and date of interview, since the year of birth is frequently reported incorrectly. In events where birth dates are not recorded or known with certainty, the mother/caregiver were probed for the approximate date of birth based on a local events calendar. The age was calculated using precise day by subtracting the date of birth from the date of data collection [8].

Every child was subjected to anthropometric measurement using standard technique and calibrated equipment. Length was measured by making child laid on flat surface, head positioned firmly against the fixed hardboard, with the eyes looking vertically. The knees extended, by applying firm pressure and feet are flexed at right angles to the lower legs on the board. Length was measured between the two boards to the nearest accuracy $0.1 \mathrm{~cm}$.

\section{Operational definitions}

\section{Stunting: (low length-for-age):}

A child was defined as stunted or chronically malnourished if the length for age index was found to be below -2 SD of the median of the standard curve. Severe stunting was diagnosed if it was below -3 SD. The length-for-age index provides an indicator of linear growth retardation and cumulative growth deficits in children. Stunting also reflects failure to receive adequate nutrition over a long period of time and is affected by recurrent and chronic illness [11].

\section{Wealth index of households:}

It was developed based on the ownership of fixed assets including radio/tape, television, table/chair, refrigerator, sofa, watch, motorcycle, mobile/telephone and others using factors analyses. The wealth index was ranked and divided into tertiles.

\section{Children's dietary diversity score:}

It was calculated asking mothers/caregivers to report the different food groups consumed by children over the past 24 hours. The Dietary diversity score (DDS) was calculated by giving a score of " 1 " for those who consumed the food item and a score of "0" for those who did not consume the food item over the past 24 hours. The DDS was then rank divided in to three subgroups (tertiles): six \& over (high), 3-5 (medium) and less than 3 (low) food groups consumed in the previous day. According to USAID $(9,22)$ the following nutritional food groups were used to calculate DDS: (1) grains, roots and tubers, (2) vitamin A-rich fruits and vegetables, (3) other fruits and vegetables, (4) meat, poultry and fish, (5) eggs, (6) pulses, legumes and nuts, (7) milk and milk products and (8) foods cooked in oil/fat/butter and sweet drinks/ foods.

\section{Data quality management}

The questionnaire was translated and contextualized to the local situation. The data were collected by Bachelor of Science (B.Sc.) degree holders who took an intensive training for three days on the questionnaire and on general approaches to data collection. Five percent (5\%) pre-test of questionnaires was done on 18 children in a similar area, which was not included in the study and some modifications were made on the basis of the findings. Measurement of length was taken in duplicate on each child. The principal investigator supervised and reviewed every questionnaire for completeness and logical consistency and made corrections on the spot.

\section{Statistical analysis}

Quantitative data were entered, coded, and analyzed using SPSS for windows version 20.0. The z-score value for Length For Age (LFA) of children generated with WHO child growth standards using WHO Anthro 2009 program, version 3.2.2 [8]. A one-sample KolmogorovSmirnov test was used to assess whether the data were normally distributed. Hosmer-Leme show test was performed for model fitness and multicollinearity also checked using variance inflation factor and correlation coefficients. Those variables that were not normally distributed were transformed log into logarithmic scale. Descriptive 
Page 3 of 7

statistics (mean $\pm \mathrm{SD}$, frequencies, proportions and tables) were used. To identify associated factors, first a bivariate logistic regression was performed for each independent variable with the outcome of interest (stunting). Finally, multivariable logistic regression was done to determine independent predictors of stunting. All tests were two-sided and $\mathrm{p}<0.05$ was considered statistically significant.

\section{Ethical Consideration}

The study was reviewed and approved by the institutional review board (IRB) of Wollega University Ethical Clearance Committee. Official letter of co-operation was also obtained from Oromia Health Bureau, Zonal Health Desk and Woredas/district Health Office. Informed verbal consent was secured from study participants in their own language after explaining the purpose of the study, potential risks and benefits of par-taking in the study and the right to withdraw from the study at any time. The participants were also assured about the confidentiality of the data. Informed consent was obtained from each participant. Confidentiality of information collected from each study participant was maintained.

\section{Results}

\section{Socio-demographic characteristics of the participants}

Five hundred ninety three mothers having children less than two years of age participated in the interview making the response rate $98.83 \%$. Two hundred ninety nine $(50.4 \%)$ of the children were males. The median age of the mother and the child were 25 years and 10 months, respectively. Majorities of mothers of the children were from Oromo ethnic group (91.1\%), $36.9 \%$ of the mothers have completed primary school, over half of the mothers were housewives, $54.6 \%$ were Protestant by religion and majority $(41.1 \%)$ of the study participants were from medium household wealth index/Socioeconomic Status (SES) (Table 1).

\begin{tabular}{|c|c|c|}
\hline Variables $(n=593)$ & & Frequency (\%) \\
\hline \multirow[t]{3}{*}{ Age of child in months } & 5-Jan & 152(25.6) \\
\hline & 11-Jun & $181(30.5$ \\
\hline & 23-Dec & $260(43.9)$ \\
\hline \multirow[t]{2}{*}{ Sex of child } & Male & $299(50.4)$ \\
\hline & Female & 294(49.6) \\
\hline \multirow[t]{5}{*}{ Age of mothers in years } & $15-19$ & $55(9.3)$ \\
\hline & $20-24$ & $224(37.8)$ \\
\hline & $25-29$ & $174(29.3)$ \\
\hline & $30-34$ & $91(15.3)$ \\
\hline & $35+$ & $49(8.3)$ \\
\hline \multirow[t]{3}{*}{ Family size } & 5-Jan & $464(78.2)$ \\
\hline & $>5$ & $129(21.8)$ \\
\hline & Mean family size & 5 \\
\hline \multirow[t]{2}{*}{ Paternal Educational status } & Illiterate & $97(16.4)$ \\
\hline & Primary complete & $205(34.6)$ \\
\hline
\end{tabular}

\begin{tabular}{|c|c|c|}
\hline & Secondary complete & $136(22.9)$ \\
\hline & Above secondary & $155(26.1)$ \\
\hline \multirow[t]{4}{*}{ Maternal Educational status } & Illiterate & $160(27)$ \\
\hline & Primary complete & $219(36.9)$ \\
\hline & Secondary complete & 113(19.1) \\
\hline & Above secondary & 101(17) \\
\hline \multirow{5}{*}{ Occupation (mothers) } & House wife & $309(52.1)$ \\
\hline & Government employee & $75(12.6)$ \\
\hline & Farmer & $18(13.7)$ \\
\hline & Merchant & $110(18.5)$ \\
\hline & Others ${ }^{*}$ & $18(3)$ \\
\hline \multirow[t]{4}{*}{ Ethnicity } & Oromo & $540(91.1)$ \\
\hline & Amahara & $41(6.9)$ \\
\hline & Guraghe & $10(1.7)$ \\
\hline & Others ${ }^{* *}$ & $2(0.3)$ \\
\hline \multirow[t]{4}{*}{ Religion } & Orthodox & $236(39.8)$ \\
\hline & Protestant & $324(54.6)$ \\
\hline & Muslim & $27(4.6)$ \\
\hline & Others $^{* * *}$ & $6(1)$ \\
\hline \multicolumn{3}{|c|}{ Wealth index of household } \\
\hline Poor & & 182(30.7) \\
\hline Medium & & $244(41.1)$ \\
\hline High & & $167(28.2)$ \\
\hline
\end{tabular}

Table 1: Socio-economic demographic characteristics of mothers of children under two years of age in rural areas of East Wollega Zone, 2014

\section{Birth order, birth weight and feeding practices}

On average about three children were ever born to a mother and of these $42 \%$ were born second and third, $38.1 \%$ were born first, $19.1 \%$ were born fourth, fifth and sixth and $0.8 \%$ were born seventh and above when categorized in their birth order.

Mothers were also asked if their baby's weight were measured at birth and $60 \%$ of the mothers reported the baby's weight were measured at birth of which majority (96.6\%) reported their baby's weight greater than or equal to 2500 grams.

The study participants were reported that majority of the children $569(96 \%)$ were ever breastfed at some point in the past and those who reported as breast fed to their baby in last 24 hours were asked the frequency of breast feeding in the last night and day time.

Three hundred ninety eight (67.1\%) of infants in the age group 0 - 6 months were exclusively breastfed in the last 24 hours of the survey. 
Citation: Wolde T, Emiru Adeba, Alemu Sufa (2014) Prevalence of Chronic Malnutrition (Stunting) and Determinant Factors among Children Aged 0-23 Months in Western Ethiopia: A Cross-Sectional Study. J Nutr Disorders Ther 4: 148. doi:10.4172/2161-0509.1000148

Page 4 of 7

Majority of the mothers (64\%) introduced complementary food to the feeding of their child at six months and about $14 \%$ of the mothers introduced complementary food at age earlier than six months (Table 2).

\begin{tabular}{|c|c|c|}
\hline Variables $(n=593)$ & & Frequency (\%) \\
\hline \multirow[t]{4}{*}{ Birth order } & 1 & $226(38.1)$ \\
\hline & 3-Feb & 249(42) \\
\hline & 6-Apr & 113(19.1) \\
\hline & $>7$ & $5(0.8)$ \\
\hline Mean of children ever born to a mother & & 2.31 \\
\hline \multirow[t]{2}{*}{ Is your baby weighted at birth? $(n=593)$} & Yes & $356(60)$ \\
\hline & No & $237(40)$ \\
\hline \multicolumn{3}{|l|}{ Birth weight of baby at birth $(n=356)$} \\
\hline$<2500$ grams & & $12(3.4)$ \\
\hline$>2500$ grams & & $344(96.6)$ \\
\hline \multicolumn{3}{|l|}{ Ever breastfeeding } \\
\hline Yes & & $569(96.0)$ \\
\hline No & & $24(4.0)$ \\
\hline \multicolumn{3}{|l|}{ Exclusive breastfeeding } \\
\hline Yes & & $398(67.1)$ \\
\hline No & & 195(32.9) \\
\hline \multicolumn{3}{|l|}{ Age started complementary foods } \\
\hline$<6$ months & & $81(13.7)$ \\
\hline At 6 months & & $378(63.7)$ \\
\hline Yet not started & & 134(22.6) \\
\hline
\end{tabular}

Table 2: Birth order, birth weight and feeding pattern of children under two years of age in rural areas of East Wollega Zone, 2014

\section{Types of food item consumed in the past 24 hours by children less than 24 months of age:}

The median intake of dietary diversity score /DDS was sixteen with the range of 10-22 and the mean \pm SD intake of dietary diversity score was 15.67 ( \pm 2.89 ). Large proportion $(39.4 \%)$ of the children were categorized in the low Dietary Diversity Score (DDS), while $30.8 \%$ and $29.8 \%$ were categorized in the medium and high dietary diversity score, respectively. In this study, the majority of the study subjects (63.6\%) of consumed foods from grain, root and tuber products, $68.2 \%$ ate foods from carrots or yellow/orange-fleshed sweet potatoes, pumpkin, ripe mango and ripe papaya, $39.3 \%$ ate foods from dark green leafy vegetables like cassava leaves \& kale, $47.6 \%$ were consumed eggs, $15.9 \%$ ate foods from meat, poultry \& fish (MPF), 34.9\% from legumes, nuts \& pulses, $40 \%$ from milk \& dairy products and $10.5 \%$ consumed from foods with oils /fats/ and sweet/soft drinks (Table 3).

\begin{tabular}{|l|l|l|}
\hline Food groups $(n=593)$ & Frequency & Per cent $(\%)$ \\
\hline
\end{tabular}

\begin{tabular}{|l|l|l|}
\hline Foods from grains, roots and tubers & 377 & 63.6 \\
\hline Any fruits \& carrots or sweet potatoes & 232 & 39.1 \\
\hline Dark green leafy vegetables & 233 & 39.3 \\
\hline MPF $^{*}$ & 94 & 15.9 \\
\hline Eggs & 282 & 47.6 \\
\hline Food made from pulses, legumes and nuts & 210 & 34.9 \\
\hline Milk and milk products & 237 & 40 \\
\hline Foods cooked with oil/fat or butter & 62 & 10.5 \\
\hline DDS Low & 164 & 39.4 \\
\hline Medium & 128 & 30.8 \\
\hline High & 124 & 29.8 \\
\hline Children diet diversity score mean \pm SD & $15.7 \pm 2.9$ & \\
\hline MPF ${ }^{*}$ Meat, poultry and fish & \multicolumn{2}{|l}{} \\
\hline
\end{tabular}

Table 3: Proportion of children 6-23 months old who consumed different food groups in the last 24 hours preceding the survey in rural areas of East Wollega Zone, 2014

\section{Prevalence of child malnutrition (stunting) in the study area}

The mean and standard deviations $( \pm \mathrm{SD})$ of the LAZ score of children 0-23 months old based on WHO Anthro software were analyzed as -0.44 and \pm 1.4 .

The prevalence of stunting (chronic malnutrition) in the study participants was $15.7 \%$. The sex specific prevalence of stunting in males was $18.1 \%$ while in females was $13.3 \%$. The age specific prevalence of stunting in age groups from 1-5 months was $20.4 \%$ and $12.2 \%$ in age groups from $6-11$ months while $15.4 \%$ was found among 12-23 months in the study area (Table 4 \& Figure 1).

\begin{tabular}{|l|l|l|}
\hline Variables (n=593) & Stunted (No \%) & Not stunted (No \%) \\
\hline Overall & $93(15.7)$ & $500(84.3)$ \\
\hline Sex & $39(13.3)$ & $255(86.7)$ \\
\hline Girls & $31(20.4)$ & $121(79.6)$ \\
\hline Age (months) & $22(12.2)$ & $159(87.8)$ \\
\hline $0-5$ & $40(15.4)$ & $220(84.6)$ \\
\hline $6-11$ &
\end{tabular}

Table 4: Prevalence of stunting by overall, sex and age groups among children under 24 months old in rural areas of East Wollega Zone, 2014 
Citation: Wolde T, Emiru Adeba, Alemu Sufa (2014) Prevalence of Chronic Malnutrition (Stunting) and Determinant Factors among Children Aged 0-23 Months in Western Ethiopia: A Cross-Sectional Study. J Nutr Disorders Ther 4: 148. doi:10.4172/2161-0509.1000148

Page 5 of 7

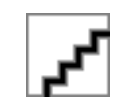

Figure 1: Length for age $\mathrm{z}$ score of children in East Wollega zone, 2014

\section{Determinants of child malnutrition (stunting)}

Table 5 shows the factors associated with stunting. Illiterate mothers had statistically significantly increased odds of being stunted compared to those literate mothers (AOR= 3.84; 95\%CI: $1.49-9.91)$. Children who consumed foods made from carrots or yellow/orangefleshed sweet potatoes and fruits were reduced odds of being stunted compared to those from non-consumer $(\mathrm{AOR}=0.51 ; 95 \% \mathrm{CI}$ : 0.28-0.95). Children who are drinking water boiled had significantly reduced odds of being stunted compared to drinking water without boiled $(\mathrm{AOR}=0.61,95 \% \mathrm{CI}: 0.39-0.97)$. High dietary diversity scores (DDS) was associated with reduced the risk of being stunted compared to low dietary diversity scores (COR $=0.51 ; 95 \% \mathrm{CI}$ : $0.26-0.99)$. Those infants who did not breast-feeding exclusively were 2 times more likely to be stunted compared with breast-fed exclusively ( $\mathrm{AOR}=2.12 ; 95 \%$ CI: 1.19-3.79).

\begin{tabular}{|c|c|c|c|c|}
\hline Variables $(n=593)$ & Stunted (n=93) No. (\%) & Not stunted ( $n=500)$ No. (\%) & Crude OR $[95 \% \mathrm{Cl}]$ & Adjusted OR $[95 \% \mathrm{Cl}]$ \\
\hline \multicolumn{5}{|c|}{ Consumed vegetables \& fruits } \\
\hline Yes & $42(18.1)$ & 190(81.9) & $0.46[0.25-0.83]^{*}$ & $0.51[0.28-0.95]^{*}$ \\
\hline No & $17(9.2)$ & 168(90.8) & 1 & 1 \\
\hline \multicolumn{5}{|c|}{ Is drinking water boiled } \\
\hline Yes & $35(20.6)$ & $135(79.4)$ & $0.76[0.31-0.89]^{*}$ & $0.61[0.39-0.97]^{*}$ \\
\hline No & $58(13.7)$ & $365(86.3)$ & 1 & 1 \\
\hline \multicolumn{5}{|l|}{ Maternal Education } \\
\hline Illiterate & $17(10.6)$ & $143(89.4)$ & $1.79[1.02-3.14]^{*}$ & $3.84[1.49-9.91]$ ** \\
\hline Literate & $76(17.6)$ & $357(82.4)$ & 1 & 1 \\
\hline \multicolumn{5}{|c|}{ Dietary diversity score } \\
\hline High & $14(11.3)$ & $110(88.7)$ & $0.51[0.26-0.99]$ * & $\ldots \ldots \ldots \ldots \ldots \ldots \ldots$ \\
\hline Medium & $12(9.4)$ & $116(90.6)$ & $1.23[0.55-2.78]$ & $\ldots \ldots \ldots \ldots \ldots \ldots \ldots$ \\
\hline Low & $33(20.1)$ & $131(79.9)$ & 1 & $\ldots \ldots \ldots \ldots \ldots \ldots \ldots$ \\
\hline \multicolumn{5}{|l|}{ Exclusive BF } \\
\hline No & $39(20)$ & $156(80)$ & & \\
\hline Yes & $54(13.6)$ & $344(86.4)$ & $1.59[1.01-2.51]^{*}$ & $2.12[1.19-3.79]]^{\star^{*}}$ \\
\hline
\end{tabular}

\section{Discussion}

This study aimed to determine the prevalence of stunting, including associated factors among children in Western Ethiopia. The prevalence of stunting in this study subjects with $15.7 \%$ of the children aged 0-23 months. This finding is very low compare with the evidence from other similar studies conducted in low- income countries including Ethiopia [5,13-16,19]. However; present finding is higher than study conducted from southern Brazil (9.1\%) [12]. This finding is also comparable with study conducted in Mongolia; the prevalence of stunting was $15.6 \%$ [18].

The prevalence of stunting was higher in children aged between 1-5 months (20.4\%) than those children aged 6-11 (12.2\%) and 12-23 (15.4\%) months. In contrast the finding of Ethiopian DHS 2011 report, the prevalence of stunting increases as the age of a child increase; with the highest prevalence of chronic malnutrition found in children age 24-35 months (57 percent) and lowest in children under age six months (10 percent) [5]. Also study conducted in Sidama zone, state that the prevalence of stunting ranged from $25 \%$ for infants aged 6-8 months to $52 \%$ for children aged $12-23$ months [6]. In this finding, prevalence of stunting higher in boys (18.1\%) than girls (13.3\%) which is support to the present finding, male children are slightly more likely to be stunted than female children (46 percent and 43 percent, respectively) [5] and $9.7 \%$ in boys while $8.4 \%$ in girls [12].

In considering some determinant factors, maternal education to be significant in this study (AOR $=3.84 ; 95 \% \mathrm{CI} 1.49-9.91)$. The association between maternal education and stunting might be attributed to the overall literacy status of the study setting in that a few of the mothers involved in the study were illiterate. In the same line, maternal education was significantly associated with chronic child undernutrition in support with the findings of other similar studies $[5,17,20-21]$. 
Page 6 of 7

Non-exclusively breastfed children were more likely to be stunted than their exclusively breastfed counterparts. This finding is consistent with similar studies conducted in developing countries including Ethiopia show that infants who are not breastfed are 6 to 10 times more likely to die in the first months of life than infants who are breastfed $[23,24]$. The study also shows that sub-optimal infant feeding practice after birth was associated with growth failure [25]. The other studies also strongly recommended that the optimal nutrition of children under two years of age, it is considered important that they be exclusively breastfed for the first 6 months before being given complementary food [26]. In many studies, it is indicated that duration of exclusive breastfeeding ( $>6$ months) and the age for starting complementary feeding is significantly associated with higher weight, length, and lower probability of stunting, wasting and infections [26-29]. This could be due to lack of essential nutrients from the breast milk during the first six months of life and later. These nutrients are known to prevent disease transmission by improving children's immunity status and through interruption of infectionmalnutrition cycle. This in turn improves child survival, growth, and development and prevents the sequel of undernutrition in later life $[30,31]$.

This study has strengths. One of its strengths is that it has used the community based approach and random selection of the study households. This may made generalization possible to the study communities as an attempt was made to identify randomized households and women with their children from the study communities. However, this study could have the following limitations. One is that the nutritional surveys are prone to Technical Error of Anthropometric Measurement (TEM), which could result in misclassification of children's nutritional status (stunting). The seasonal variation of food availability that will have an effect on dietary diversity intake was not considered due to the cross sectional nature of the study. Furthermore, it was difficult to establish a cause-effect relationship between the dependent variable (stunting) and the independent variables though association was observed in West Ethiopia.

\section{Conclusions and Recommendations}

The study showed although the prevalence rate of stunting in the study area was found lower than some reported elsewhere, still there is an indicator of childhood chronic malnutrition remains a major public health problem in Western Ethiopia. Stunting was related to the illiterate mothers and non-exclusive breastfeeding practice. Thus, an organized effort should be made at all levels to improve maternal education and exclusive breastfeeding practice of the poor rural population particularly mothers to curb the problems of chronic undernutrition (stunting) in children, especially in the first two years of life.

\section{Authors' Contributions}

TW conceived and designed the study, performed analysis and interpretation of data and drafted the manuscript. EA assisted with the design conception, analysis and interpretation of data, and the critical review of the manuscript. AS assisted the study design, data interpretation, and critically reviewed the manuscript. All authors read and approved the final manuscript.

\section{Acknowledgments}

The authors would like to thank Wollega University for the technical and financial support. The study participants and all individuals who render help during the study are highly acknowledged.

\section{References}

1. World Health organization (2010) Global Data Bank on Infant and Young Child Feeding, World Health Statistics. Geneva, World Health Organization.

2. World Health organization (2001) Guiding Principle for Complementary feeding of Breastfed Child. WHO.

3. Martorell eta'l, R. J. (2009). Long-term consequences of growth retardation during early childhood. Amer J of food and Agr.

4. Federal Ministry of health (FMOH) (2004) National strategy for Infant and young child feeding in Ethiopia. Addis Ababa, Ethiopia.

5. Centrla Statistic Authority (CSA) (2011) Ethiopia Demographic and Health Survey. Addis Ababa: Central Statistic Authority.

6. Rosalind S. Gibson, Yewelsew Abebe, K. Michael Hambidge, Isabel Arbide, Aklilu Teshome, et al. (2009) Inadequate feeding practices and impaired growth among children from subsistence farming households in Sidama, Southern Ethiopia. J. Maternal and Child Nutrition 5:275.

7. FDRE C (2008). Summary and statistical report of the 2007 population and Housing census. Addis Ababa.

8. WHO (World Health Organization) (2009) WHO AnthroPlus for personal computers Manual: Software for assessing growth of the world's children and adolescents. Geneva: Switzerland.

9. Swindale A. \& Bilinsky (2006) Household dietary diversity score (HDDS) for measurement of household food access: Indicator guide (v.2). Food and Nutrition Technical Assistance Project, Academy for Educational Development 1-9.

10. Gibson RS. (2005) Principles of Nutritional Assessment.2nd ed. Oxford University Press, New York 275-276.

11. WHO Multicenter Growth Reference Study Group (2006) WHO Child Growth Standards: Length/ height-for-age, weight-for-age, weight-forlength, weight-for-height and body mass index-for-age: Methods and development. Geneva, World Health Organization.

12. Vitolo MR1, Gama CM, Bortolini GA, Campagnolo PD, Drachler Mde L (2008) Some risk factors associated with overweight, stunting and wasting among children under 5 years old. J Pediatr (Rio J) 84: 251-257.

13. Zewdu S (2012) Magnitude and Factors Associated with Malnutrition of Children Under Five Years of Age in Rural Kebeles of Haramaya, Ethiopia. Harar Bulletin of Health Sciences 4:221-232.Tesfaye M. (2009) Bayesian approach to identify predictors of children Nutritional status in Ethiopia.

14. Mengistu K, Alemu K, Destaw B (2013) Prevalence of Malnutrition and Associated Factors Among Children Aged 6-59 Months at HidabuAbote District, North Shewa, Oromia Regional State. J Nutr Disorders Ther T1: 001.doi:10.4172/2161-0509.T1-001.

15. Kebede E (2007) Prevalence and Determinants of Child Malnutrition in Gimbi district Pp27-39.

16. Edris $M(2006)$ Assessment of nutritional status of preschool children of Gumbrit, Ethiop J Health Dev 21: 125-129.

17. Paudel R1, Pradhan B, Wagle RR, Pahari DP, Onta SR (2012) Risk factors for stunting among children: a community based case control study in Nepal. Kathmandu Univ Med J (KUMJ) 10: 18-24.

18. Otgonjargal D, Bradley A, Woodruff F, Batjargal, et al.(2012) Nutritional status of under- five children in Mongolia. Journal of Medicine and Medical Sciences 3: 341-349.

19. Sapkota VP, Gurung CK (2009) Prevalence and Predictors of Underweight, Stunting and Wasting in Under-Five Children. Nepal Health Res Counc. October, 7: 120-126. 
Citation: Wolde T, Emiru Adeba, Alemu Sufa (2014) Prevalence of Chronic Malnutrition (Stunting) and Determinant Factors among Children Aged 0-23 Months in Western Ethiopia: A Cross-Sectional Study. J Nutr Disorders Ther 4: 148. doi:10.4172/2161-0509.1000148

Page 7 of 7

20. Wamani H1, Astrøm AN, Peterson S, Tumwine JK, Tylleskar T (2006) Predictors of poor anthropometric status among children under 2 years of age in rural Uganda. Public Health Nutr 9: 320-326.

21. Semba RD1, de Pee S, Sun K, Sari M, Akhter N, et al. (2008) Effect of parental formal education on risk of child stunting in Indonesia and Bangladesh: a cross-sectional study. Lancet 371: 322-328.

22. Hatløy A1, Hallund J, Diarra MM, Oshaug A (2000) Food variety, socioeconomic status and nutritional status in urban and rural areas in Koutiala (Mali). Public Health Nutr 3: 57-65.

23. UNICEF (2007) Progress for children: a world fit for children. Statistical Review Number 6. New York, UNICEF.

24. Bahl R1, Frost C, Kirkwood BR, Edmond K, Martines J, et al. (2005) Infant feeding patterns and risks of death and hospitalization in the first half of infancy: multicentre cohort study. Bull World Health Organ 83: 418-426.

25. Engebretsen IM1, Tylleskär T, Wamani H, Karamagi C, Tumwine JK (2008) Determinants of infant growth in Eastern Uganda: a communitybased cross-sectional study. BMC Public Health 8: 418.
26. Kuchenbecker J, Jordan I, Reinbott A, Herrmann J, Jeremias T, et al. (2014) Exclusive breastfeeding and its effect on growth of Malawian infants: results from a cross-sectional study. Paediatr Int Child Health .

27. Jiang Y1, Su X, Wang C, Zhang L, Zhang X, et al. (2014) Prevalence and risk factors for stunting and severe stunting among children under three years old in mid-western rural areas of China. Child Care Health Dev .

28. Mason JB1, Shrimpton R2, Saldanha LS2, Ramakrishnan U3, Victora CG4, et al. (2014) The first 500 days of life: policies to support maternal nutrition. Glob Health Action 7: 23623.

29. Haschke F1, Haiden N, Detzel P, Yarnoff B, Allaire B, et al. (2013) Feeding patterns during the first 2 years and health outcome. Ann Nutr Metab 62 Suppl 3: 16-25.

30. WHO (2008) Learning From Large-Scale Community-Based Programmes: To Improve BreastfeedingPractices.

31. Al-Sahab B1, Lanes A, Feldman M, Tamim H (2010) Prevalence and predictors of 6-month exclusive breastfeeding among Canadian women: a national survey. BMC Pediatr 10: 20. 